PROCEEDINGS OF THE

AMERICAN MATHEMATICAL SOCIETY

Volume 135, Number 8, August 2007, Pages 2539-2547

S 0002-9939(07)08756-4

Article electronically published on March 21, 2007

\title{
LINEAR BIJECTIONS PRESERVING THE HÖLDER SEMINORM
}

\author{
A. JIMÉNEZ-VARGAS
}

(Communicated by N. Tomczak-Jaegermann)

\begin{abstract}
Let $(X, d)$ be a compact metric space and let $\alpha$ be a real number with $0<\alpha<1$. The aim of this paper is to solve a linear preserver problem on the Banach algebra $C^{\alpha}(X)$ of Hölder functions of order $\alpha$ from $X$ into $\mathbb{K}$. We show that each linear bijection $T: C^{\alpha}(X) \rightarrow C^{\alpha}(X)$ having the property that $\alpha(T(f))=\alpha(f)$ for every $f \in C^{\alpha}(X)$, where

$$
\alpha(f)=\sup \left\{\frac{|f(x)-f(y)|}{d^{\alpha}(x, y)}: x, y \in X, x \neq y\right\},
$$

is of the form $T(f)=\tau f \circ \varphi+\mu(f) 1_{X}$ for every $f \in C^{\alpha}(X)$, where $\tau \in \mathbb{K}$ with $|\tau|=1, \varphi: X \rightarrow X$ is a surjective isometry and $\mu: C^{\alpha}(X) \rightarrow \mathbb{K}$ is a linear functional.
\end{abstract}

\section{INTRODUCTION}

Let $(X, d)$ be a compact metric space and let $\alpha$ be a real number with $0<\alpha<1$. A function $f: X \rightarrow \mathbb{K}$ is said to be a Hölder function of order $\alpha$ if there is a constant $k$ such that

$$
|f(x)-f(y)| \leq k d^{\alpha}(x, y), \quad \forall x, y \in X .
$$

The smallest constant $k$ for which the above inequality holds is called the Hölder seminorm of order $\alpha$ of $f$ and it is denoted by $\alpha(f)$, that is,

$$
\alpha(f)=\sup \left\{\frac{|f(x)-f(y)|}{d^{\alpha}(x, y)}: x, y \in X, x \neq y\right\} .
$$

Let $C^{\alpha}(X)$ be the vector space of all the functions $f$ from $X$ into $\mathbb{K}$ such that $\alpha(f)$ is finite. This supremum does not define a norm on $C^{\alpha}(X)$, since $\alpha(f)=0$ if and only if $f$ is constant on $X$.

It is said that a linear bijection $T: C^{\alpha}(X) \rightarrow C^{\alpha}(X)$ preserves the Hölder seminorm if

$$
\alpha(T(f))=\alpha(f), \quad \forall f \in C^{\alpha}(X) .
$$

By brevity, these maps will be called Hölder seminorm preserving.

The most common way to actually obtain a norm on $C^{\alpha}(X)$ is to define

$$
\|f\|=\max \left\{\alpha(f),\|f\|_{\infty}\right\},
$$

Received by the editors January 10, 2006 and, in revised form, February 13, 2006 and April $11,2006$.

2000 Mathematics Subject Classification. Primary 46E15; Secondary 46J10.

Key words and phrases. Linear preserver problem, extreme point, isometry.

This research was supported by Junta de Andalucia project P06-FQM-01438.

(C)2007 American Mathematical Society Reverts to public domain 28 years from publication 
where

$$
\|f\|_{\infty}=\sup \{|f(x)|: x \in X\} .
$$

An advantage of this approach is that the Banach space of Hölder functions obtained is actually a Banach algebra. This space of functions and other closely related spaces have been the subject of considerable study (see, for example, [4, 5, 6, 9, 10, 11]).

Linear preserver problems concern the question of determining all linear maps on algebras of matrices or operators which leave invariant a given set, function or relation (see, for example, the survey papers [7, 8]), but similar questions can be raised on arbitrary algebras.

Let $Y$ be a compact Hausdorff space and let $C(Y)$ be the space of real or complex continuous functions on $Y$ equipped with the supremum norm. With regard to $C(Y)$, the main linear preserver problems concern the characterization of linear bijections preserving some given seminorm or norm. The classical Banach-Stone theorem determines the linear bijections preserving the sup-norm on $C(Y)$. Recently, when $Y$ is a first countable space, Györy and Molnar [3] have given a complete description of linear bijections of $C(Y)$ which preserve the seminorm

$$
f \mapsto \operatorname{diam}(f(Y))=\sup \{|f(x)-f(y)|: x, y \in Y\} .
$$

Their result has been extended to the case of a general compact Hausdorff space by González and Uspenskij [2] and independently by Cabello Sánchez [1].

Motivated by this, the purpose of this paper is to determine all Hölder seminorm preserving linear bijections of $C^{\alpha}(X)$.

\section{The Linear BIJECTIONS Which PRESERVE THE HölDER SEMINORM}

Our characterization of these maps is the following.

Theorem 2.1. A linear bijection $T: C^{\alpha}(X) \rightarrow C^{\alpha}(X)$ is Hölder seminorm preserving if and only if there is a surjective isometry $\varphi: X \rightarrow X$, a linear functional $\mu: C^{\alpha}(X) \rightarrow \mathbb{K}$ and a number $\tau$ with $|\tau|=1$ and $\mu\left(1_{X}\right)+\tau \neq 0$ such that $T(f)=\tau f \circ \varphi+\mu(f) 1_{X}$ for every $f \in C^{\alpha}(X)$.

To prove Theorem 2.1, we follow a similar process to the one of Félix Cabello Sánchez in 11. Our approach depends on the analysis of the isometry group of certain Banach spaces of Hölder functions in which the Hölder seminorm becomes a norm.

There is another way to obtain a norm on the vector space of Hölder functions, and that is to identify a distinguished point of the metric space and consider only the functions which are zero at this point.

Let $x_{0}$ be an arbitrarily chosen point of $X$. The set $C^{\alpha}\left(X, x_{0}\right)$ of all the functions $f \in C^{\alpha}(X)$ such that $f\left(x_{0}\right)=0$ becomes a Banach space endowed with the norm $f \mapsto \alpha(f)$. The map $\rho: C^{\alpha}(X) \rightarrow C^{\alpha}\left(X, x_{0}\right)$ defined by

$$
\rho(f)=f-f\left(x_{0}\right) 1_{X}, \quad \forall f \in C^{\alpha}(X),
$$

is surjective linear with $\operatorname{ker} \rho$ equal to the space of the constant functions on $X$ and

$$
\alpha(\rho(f))=\alpha(f), \quad \forall f \in C^{\alpha}(X) .
$$


Suppose that $T$ is a Hölder seminorm preserving linear bijection of $C^{\alpha}(X)$. Then there is a (unique) surjective linear isometry $T_{\alpha}$ of $C^{\alpha}\left(X, x_{0}\right)$ such that the diagram

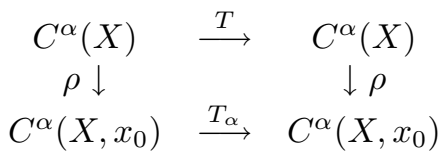

commutes.

We derive Theorem 2.1 from the following characterization of the isometries of $C^{\alpha}\left(X, x_{0}\right)$. We shall denote by $S_{\mathbb{K}}$ the set of all the unimodular scalars of $\mathbb{K}$.

Theorem 2.2. A linear map $T_{\alpha}: C^{\alpha}\left(X, x_{0}\right) \rightarrow C^{\alpha}\left(X, x_{0}\right)$ is a surjective isometry if and only if there is a surjective isometry $\varphi$ of $X$ and a number $\tau$ in $S_{\mathbb{K}}$ such that $T_{\alpha}(\rho(f))=\rho(\tau f \circ \varphi)$ for all $f \in C^{\alpha}(X)$.

Proof of Theorem 2.1. It is straightforward to check that every linear map $T$ of the form $T(f)=\tau f \circ \varphi+\mu(f) 1_{X}$ for every $f \in C^{\alpha}(X)$ with $\tau, \varphi, \mu$ being as in Theorem 2.1 , is a Hölder seminorm preserving linear bijection of $C^{\alpha}(X)$.

Now, suppose that $T: C^{\alpha}(X) \rightarrow C^{\alpha}(X)$ is a linear bijection which preserves the Hölder seminorm and let $T_{\alpha}$ be the corresponding isometry of $C^{\alpha}\left(X, x_{0}\right)$ such that $T_{\alpha} \circ \rho=\rho \circ T$. By Theorem 2.2 there is a surjective isometry $\varphi$ of $X$ and a number $\tau \in S_{\mathbb{K}}$ such that $T_{\alpha}(\rho(f))=\rho(\tau f \circ \varphi)$ for all $f \in C^{\alpha}(X)$. Then $T(f)-\tau f \circ \varphi \in \operatorname{ker} \rho$ for all $f \in C^{\alpha}(X)$, and therefore there exists a linear functional $\mu: C^{\alpha}(X) \rightarrow \mathbb{K}$ such that $T(f)=\tau f \circ \varphi+\mu(f) 1_{X}$ for every $f \in C^{\alpha}(X)$. Since the relation $\mu\left(1_{X}\right) \neq$ $-\tau$ is obvious, the proof of Theorem 2.1 is complete.

For the proof of Theorem 2.2 we shall need a description of the following points.

\section{The extreme points of the unit ball of $C^{\alpha}\left(X, x_{0}\right)^{*}$}

These extreme points play a key role in our characterization of the isometries of $C^{\alpha}\left(X, x_{0}\right)$. To identify them, we construct a linear isometric imbedding of $C^{\alpha}\left(X, x_{0}\right)$ into a suitable space of continuous functions supplied with the supremum norm.

Let $W$ be the complement of the diagonal in the cartesian product space $X \times X$, that is, $W=\{(x, y) \in X \times X: x \neq y\}$ and let $\beta W$ be the Stone-Cech compactification of $W$.

If $C(\beta W)$ denotes the Banach space of continuous functions from $\beta W$ into $\mathbb{K}$ endowed with the supremum norm, we define the map $r: C^{\alpha}\left(X, x_{0}\right) \rightarrow C(\beta W)$ by

$$
r(f)(w)=\beta f^{*}(w), \quad \forall f \in C^{\alpha}\left(X, x_{0}\right), \forall w \in \beta W,
$$

where

$$
f^{*}(x, y)=\frac{f(x)-f(y)}{d^{\alpha}(x, y)}, \quad \forall(x, y) \in W
$$

and $\beta f^{*}$ is its norm-preserving extension to $\beta W$. Clearly $r$ is linear and

$$
\|r(f)\|_{\infty}=\left\|\beta f^{*}\right\|_{\infty}=\left\|f^{*}\right\|_{\infty}=\alpha(f)
$$

for every $f \in C^{\alpha}\left(X, x_{0}\right)$.

For every $w \in \beta W$, we shall denote by $\delta_{w}$ the evaluation functional on $C(\beta W)$ given by $\delta_{w}(f)=f(w)$. Moreover, $\widetilde{\delta}_{w}$ will stand for the functional $\delta_{w} \circ r$, that is,

$$
\widetilde{\delta}_{w}(f)=\delta_{w}(r(f)), \quad \forall f \in C^{\alpha}\left(X, x_{0}\right) .
$$


Clearly $\widetilde{\delta}_{w}$ is linear and

$$
\left|\widetilde{\delta}_{w}(f)\right|=\left|\beta f^{*}(w)\right| \leq\left\|\beta f^{*}\right\|_{\infty}=\alpha(f), \quad \forall f \in C^{\alpha}\left(X, x_{0}\right) .
$$

Hence $\widetilde{\delta}_{w} \in C^{\alpha}\left(X, x_{0}\right)^{*}$ and $\left\|\widetilde{\delta}_{w}\right\| \leq 1$.

Lemma 3.1. Each extreme point of the unit ball of $C^{\alpha}\left(X, x_{0}\right)^{*}$ is of the form $\tau \widetilde{\delta}_{w}$ for some $\tau \in S_{\mathbb{K}}$ and some $w \in \beta W$.

Proof. The linear map $r$ imbeds $C^{\alpha}\left(X, x_{0}\right)$ isometrically as a subspace of $C(\beta W)$. As a consequence, the adjoint map $r^{*}: C(\beta W)^{*} \rightarrow C^{\alpha}\left(X, x_{0}\right)^{*}$ sends the unit ball of $C(\beta W)^{*}$ onto the unit ball of $C^{\alpha}\left(X, x_{0}\right)^{*}$. Thus the Krein-Milman Theorem implies that each extreme point of the unit ball of $C^{\alpha}\left(X, x_{0}\right)^{*}$ is the image under $r^{*}$ of some extreme point of the unit ball of $C(\beta W)^{*}$. By the Arens-Kelley Theorem, the extreme points of the unit ball of $C(\beta W)^{*}$ are of the form $\tau \delta_{w}$ where $\tau \in S_{\mathbb{K}}$ and $w \in \beta W$.

Therefore, if $\Phi$ is an extreme point of the unit ball of $C^{\alpha}\left(X, x_{0}\right)^{*}$, we have

$$
\Phi=r^{*}\left(\tau \delta_{w}\right)=\tau r^{*}\left(\delta_{w}\right)=\tau\left(\delta_{w} \circ r\right)=\tau \widetilde{\delta}_{w}
$$

for some $\tau \in S_{\mathbb{K}}$ and some $w \in \beta W$.

Observe that for each $(x, y)$ in $W$, the functional $\widetilde{\delta}_{(x, y)}$ comes given by

$$
\widetilde{\delta}_{(x, y)}(f)=\delta_{(x, y)}(r(f))=\beta f^{*}(x, y)=f^{*}(x, y)=\frac{f(x)-f(y)}{d^{\alpha}(x, y)}
$$

for every $f \in C^{\alpha}\left(X, x_{0}\right)$.

Next we shall study the relations between the extreme points of the unit ball of $C^{\alpha}\left(X, x_{0}\right)^{*}$ and the functionals of the form $\widetilde{\delta}_{(x, y)}$ with $(x, y) \in W$. Mayer-Wolf states these relations in 9] without proof (see remark after Theorem 2.3). We include the proofs for the sake of completeness, but we shall detail essentially those steps which do not appear in 9 .

We first prove that every functional $\widetilde{\delta}_{(x, y)}$ is an extreme point of the unit ball of $C^{\alpha}\left(X, x_{0}\right)^{*}$. We shall need the following technical lemma ([12], Lemma 2.4.4).

Lemma 3.2. Let $0<\alpha<\beta<1$ and let $A, \varepsilon>0$. Then there exists $\delta>0$ such that for any $a, b, c, d \geq 0$ and $B>0$ which satisfy the three conditions:

$$
\begin{gathered}
|a-b|,|c-d| \leq A \leq a+b, c+d, \\
|b-c|,|a-d| \leq B \leq b+c, a+d, \\
\varepsilon \leq a+c, b+d,
\end{gathered}
$$

we have

$$
\frac{\left|a^{\beta}-b^{\beta}+c^{\beta}-d^{\beta}\right|}{2 A^{\beta-\alpha} B^{\alpha}} \leq 1-\delta .
$$

Lemma 3.3. Let $\tau$ be in $S_{\mathbb{K}}$ and $(x, y)$ in $W$. Then $\tau \widetilde{\delta}_{(x, y)}$ is an extreme point of the unit ball of $C^{\alpha}\left(X, x_{0}\right)^{*}$.

Proof. The proof that $\tau \widetilde{\delta}_{(x, y)}$ (or $\widetilde{\delta}_{(x, y)}$ equivalently) is an extreme point of the unit ball of $C^{\alpha}\left(X, x_{0}\right)^{*}$ will be based on the following fact which is a special case of a more general result proved by de Leeuw ([6], see Lemma 3.2):

Let $X$ be a compact Hausdorff space and $A$ a closed subspace of $C(X)$. If $x \in X$ and $\delta_{x}$ is the evaluation functional $f \in C(X) \rightarrow f(x) \in \mathbb{K}$, a sufficient condition 
for $\left.\delta_{x}\right|_{A}$ to be an extreme point of the unit ball of $A^{*}$ is that there exists a function $f$ in the unit ball of $A$ such that $f(x)=1$, and $|f(y)|=1$ iff there exists a $\theta= \pm 1$ such that $g(y)=\theta g(x)$ for all $g \in A$. In this case we say that $f$ peaks at $x$ relative to $A$.

Taking into account this result, we only have to find a function $f_{(x, y)} \in C^{\alpha}\left(X, x_{0}\right)$ such that $r\left(f_{(x, y)}\right)$ peaks at $(x, y)$ relative to $r\left(C^{\alpha}\left(X, x_{0}\right)\right)$. To see this we follow (12, Proposition 2.4.5). Choose a real number $\beta$ with $\alpha<\beta<1$ and consider the function $f_{(x, y)}=\rho\left(g_{(x, y)}\right)$, where

$$
g_{(x, y)}(z)=\frac{d^{\beta}(z, y)-d^{\beta}(z, x)}{2 d^{\beta-\alpha}(x, y)}, \quad \forall z \in X
$$

Clearly

$$
r\left(f_{(x, y)}\right)(x, y)=\frac{f_{(x, y)}(x)-f_{(x, y)}(y)}{d^{\alpha}(x, y)}=1 .
$$

Let $U$ be any open subset of $W$ (in the topology induced by the sup metric) containing $(x, y)$ and $(y, x)$. Then there exists $\varepsilon>0$ such that

$$
(z, w) \notin U \quad \Rightarrow \quad d(x, z)+d(y, w) \geq \varepsilon, d(x, w)+d(y, z) \geq \varepsilon .
$$

For this $\varepsilon$ and $A=d(x, y)$, let $\delta$ be given by the above lemma. For any $(z, w) \notin U$, let $a=d(y, z), b=d(x, z), c=d(x, w), d=d(y, w)$ and $B=d(z, w)$. A simple calculation shows that the hypotheses of the lemma are satisfied and thus we have

$$
\left|r\left(f_{(x, y)}\right)(z, w)\right|=\frac{\left|f_{(x, y)}(z)-f_{(x, y)}(w)\right|}{d^{\alpha}(z, w)}=\frac{\left|a^{\beta}-b^{\beta}+c^{\beta}-d^{\beta}\right|}{2 A^{\beta-\alpha} B^{\alpha}} \leq 1-\delta .
$$

This shows that $f_{(x, y)} \in C^{\alpha}\left(X, x_{0}\right)$ with

$$
\left\|r\left(f_{(x, y)}\right)\right\|_{\infty}=\alpha\left(f_{(x, y)}\right)=1,
$$

and $\left|r\left(f_{(x, y)}\right)(w)\right|<1$ for all $w \in \beta W$ except at $w=(x, y)$ and $w=(y, x)$. According to the definition, $r\left(f_{(x, y)}\right)$ peaks at $(x, y)$ relative to $r\left(C^{\alpha}\left(X, x_{0}\right)\right)$.

Let $\Delta: W \rightarrow C^{\alpha}\left(X, x_{0}\right)^{*}$ be the map defined by

$$
\Delta(x, y)=\widetilde{\delta}_{(x, y)} .
$$

Lemma 3.4. The map $\Delta: W \rightarrow\left(\Delta(W), w^{*}\right)$ is a local homeomorphism.

Proof. The proof will be carried out through a series of steps.

Step 1. Let $\left(x_{1}, y_{1}\right),\left(x_{2}, y_{2}\right)$ be in $W$ and suppose that $\widetilde{\delta}_{\left(x_{1}, y_{1}\right)}=\widetilde{\delta}_{\left(x_{2}, y_{2}\right)}$. Then $\left\{x_{1}, y_{1}\right\}=\left\{x_{2}, y_{2}\right\}$.

Proof. Let us assume, to obtain a contradiction, that $\left\{x_{1}, y_{1}\right\} \neq\left\{x_{2}, y_{2}\right\}$. Then there exists at least a point in $\left\{x_{2}, y_{2}\right\}$ which is not in $\left\{x_{1}, y_{1}\right\}$. We can suppose, without loss of generality, that such a point is $x_{2}$. Let $g: X \rightarrow \mathbb{R}$ be the function defined by

$$
g(z)=\frac{d^{\alpha}\left(z,\left\{x_{1}, y_{1}, y_{2}\right\}\right)}{d^{\alpha}\left(z, x_{2}\right)+d^{\alpha}\left(z,\left\{x_{1}, y_{1}, y_{2}\right\}\right)}, \quad \forall z \in X .
$$

It is easy to check that $g \in C^{\alpha}(X)$. Therefore the function $f=\rho(g) \in C^{\alpha}\left(X, x_{0}\right)$ and for every $(x, y) \in W$, it is clear that

$$
\widetilde{\delta}_{(x, y)}(f)=\frac{g(x)-g(y)}{d^{\alpha}(x, y)} .
$$


In particular we obtain that $\widetilde{\delta}_{\left(x_{1}, y_{1}\right)}(f)=0$ and $\widetilde{\delta}_{\left(x_{2}, y_{2}\right)}(f)=\frac{1}{d^{\alpha}\left(x_{2}, y_{2}\right)}$. Since $\widetilde{\delta}_{\left(x_{1}, y_{1}\right)}=\widetilde{\delta}_{\left(x_{2}, y_{2}\right)}$, a contradiction follows. So $\left\{x_{1}, y_{1}\right\}=\left\{x_{2}, y_{2}\right\}$.

Step 2. Given $(x, y)$ in $W$, there exists a neighbourhood $B_{\varepsilon}(x, y)$ of $(x, y)$ in $W$ for a suitable $\varepsilon>0$ such that $(w, z) \notin B_{\varepsilon}(x, y)$ if $(z, w) \in B_{\varepsilon}(x, y)$.

Proof. For each $\varepsilon>0$ the set

$$
B_{\varepsilon}(x, y)=\left\{(z, w) \in W: d^{\alpha}(z, x)<\varepsilon, d^{\alpha}(w, y)<\varepsilon\right\}
$$

is a neighbourhood of $(x, y)$ in $W$ with the topology induced by the sup metric. Let $\varepsilon$ be a real number such that $0<\varepsilon<d^{\alpha}(x, y) / 2$. Then the neighbourhoods $B_{\varepsilon}(x, y)$ and $B_{\varepsilon}(y, x)$ are disjoint. If $(z, w) \in B_{\varepsilon}(x, y)$, then clearly $(w, z) \in B_{\varepsilon}(y, x)$ and therefore $(w, z) \notin B_{\varepsilon}(x, y)$.

Step 3. Given $(x, y)$ in $W$, the map $\Delta$ is injective on $B_{\varepsilon}(x, y)$.

Proof. This follows immediately from Steps 1 and 2.

Step 4. The map $\Delta: W \rightarrow\left(\Delta(W), w^{*}\right)$ is continuous.

Proof. Let $(x, y) \in W$. If $\left\{\left(x_{i}, y_{i}\right)\right\}$ is a net in $W$ converging to $(x, y)$, then $\left\{f\left(x_{i}, y_{i}\right)\right\}$ converges to $f(x, y)$ for every $f$ in $C(\beta W)$. This says that $\left\{\delta_{\left(x_{i}, y_{i}\right)}\right\}$ converges to $\delta_{(x, y)}$ in $\left(C(\beta W)^{*}, w^{*}\right)$. Since $r^{*}$ (as an adjoint mapping) is continuous from $\left(C(\beta W)^{*}, w^{*}\right)$ into $\left(C^{\alpha}\left(X, x_{0}\right)^{*}, w^{*}\right)$, then $\left\{r^{*}\left(\delta_{\left(x_{i}, y_{i}\right)}\right)\right\}=\left\{\widetilde{\delta}_{\left(x_{i}, y_{i}\right)}\right\}$ converges to $r^{*}\left(\delta_{(x, y)}\right)=\widetilde{\delta}_{(x, y)}$ in $\left(C^{\alpha}\left(X, x_{0}\right)^{*}, w^{*}\right)$.

Step 5. The map $\Delta: W \rightarrow\left(\Delta(W), w^{*}\right)$ is open.

Proof. Let $U$ be an open subset of $W$ and let $(x, y) \in U$. We take a suitable neighbourhood $B_{\varepsilon}(x, y) \subset U$ and we can find a function $f \in C^{\alpha}\left(X, x_{0}\right)$ such that $r(f)(x, y)=1$ and $r(f)(z, w)=0$ for all $(z, w)$ in $W-B_{\varepsilon}(x, y)$. Let $V_{1}=$ $\left\{\Phi \in C^{\alpha}\left(X, x_{0}\right)^{*}: \Phi(f) \neq 0\right\}$. Then $V_{1}$ is $w^{*}$-open in $C^{\alpha}\left(X, x_{0}\right)^{*}$. Hence $V_{1} \cap \Delta(W)$ is $w^{*}$-open in $\Delta(W)$ and we have that $\widetilde{\delta}_{(x, y)} \in V_{1} \cap \Delta(W) \subset \Delta\left(B_{\varepsilon}(x, y)\right) \subset \Delta(U)$.

Step 6. Given $(x, y)$ in $W$, the map $\Delta$ is a homeomorphism from $B_{\varepsilon}(x, y)$ into $\left(\Delta\left(B_{\varepsilon}(x, y)\right), w^{*}\right)$.

Proof. The proof is deduced from the above steps.

From now on $F(W)$ stands for the set

$$
\left\{\tau \widetilde{\delta}_{(x, y)}: \tau \in S_{\mathbb{K}},(x, y) \in W\right\} .
$$

Every element of $F(W)$ is an extreme point of the unit ball of $C^{\alpha}\left(X, x_{0}\right)^{*}$, but the reciprocal is not true. However we have the following fact:

Lemma 3.5. Every extreme point of the unit ball of $C^{\alpha}\left(X, x_{0}\right)^{*}$ belongs to the $w^{*}$-closure of $F(W)$.

Proof. Let $\Phi$ be an extreme point of the unit ball of $C^{\alpha}\left(X, x_{0}\right)^{*}$. Then $\Phi=\tau \widetilde{\delta}_{w}$ for some $\tau \in S_{\mathbb{K}}$ and some $w \in \beta W$ by Lemma 3.1. If $w \in W$, there is nothing to prove. If $w \in \beta W \backslash W$, since $W$ is dense in $\beta W$, we can select a net $\left\{w_{i}\right\}$ from $W$ converging to $w$ in $\beta W$. Reasoning as in the proof of Step 4 in Lemma 3.4, we show that $\left\{\widetilde{\delta}_{w_{i}}\right\}$ converges to $\widetilde{\delta}_{w}$ in $\left(C\left(X, x_{0}\right)^{*}, w^{*}\right)$ and so $\Phi$ is in the $w^{*}$-closure of $F(W)$. 
A complete description of the extreme points of the unit ball of $C^{\alpha}\left(X, x_{0}\right)^{*}$ of the form $\widetilde{\delta}_{w}$ with $w \in \beta W \backslash W$ appears difficult ([5], see page 185). For example, Johnson (4], Theorem 2.8) states that if the compact metric space $X$ is an infinite set, then the unit ball of the space $C^{\alpha}(X)^{*}$ has extreme points of this type, and Sherbert ([10, Chapter III) shows that these functionals must be point derivations.

However the $w^{*}$-topology supplies a property which permits us to distinguish the extreme points of the form $\widetilde{\delta}_{(x, y)}$ with $(x, y) \in W$ from those which are of the form $\widetilde{\delta}_{w}$ with $w \in \beta W \backslash W$.

Lemma 3.6. Let $\Phi$ be an extreme point of the unit ball of $C^{\alpha}\left(X, x_{0}\right)^{*}$. Then $\Phi$ belongs to $F(W)$ if and only if $\Phi$ has a $w^{*}$-metrizable neighbourhood in the $w^{*}$ closure of $F(W)$.

Proof. The proof of the necessity is immediate since $\left(\Delta(W), w^{*}\right)$ and $W$ are locally homeomorphic by Lemma 3.4.

To prove the sufficiency suppose that $\Phi \notin F(W)$ and that $\Phi$ has a $w^{*}$-metrizable neighbourhood in the $w^{*}$-closure of $F(W)$. The existence of this neighbourhood implies that there is a sequence $\left\{\Phi_{n}\right\}$ in $F(W)$ such that $\left\{\Phi_{n}\right\}$ converges to $\Phi$ in the $w^{*}$-topology.

Since $\Phi \notin F(W)$, then $\Phi=\tau \widetilde{\delta}_{w}$ for some $\tau \in S_{\mathbb{K}}$ and $w \in \beta W \backslash W$ by Lemma 3.1. Since $W$ is dense in $\beta W$, there is a net $\left\{w_{i}\right\}=\left\{\left(x_{i}, y_{i}\right)\right\}$ in $W$ converging to $w$ in $\beta W$. By ([10, Lemma 9.6) the nets $\left\{x_{i}\right\}$ and $\left\{y_{i}\right\}$ converge in $X$ to the same point $x_{w} \in X$ and this point $x_{w}$ is independent of the choice of net $\left\{w_{i}\right\}$ from $W$.

In view of these comments we can suppose without loss of generality that $\Phi_{n}=$ $\tau \widetilde{\delta}_{\left(x_{n}, y_{n}\right)}$ with $\left\{x_{n}\right\}$ and $\left\{y_{n}\right\}$ converging to $x_{w}$. Following the proof of (9], Theorem $2.3)$, we construct a function $f \in C^{\alpha}\left(X, x_{0}\right)$ such that $\lim _{n \rightarrow \infty} \widetilde{\delta}_{\left(x_{n}, y_{n}\right)}(f)$ does not exist and so we obtain a contradiction.

\section{The isometry group of $C^{\alpha}\left(X, x_{0}\right)$}

Now we are ready to characterize the isometries of $C^{\alpha}\left(X, x_{0}\right)$. The isometries between pairs of spaces of this type have been studied in 9 .

Proof of Theorem 2.2. It is easy to verify that the map $T$ of the form $T(\rho(f))=$ $\rho(\tau f \circ \varphi)$ for all $f \in C^{\alpha}(X)$ with $\tau, \varphi$ under the assumptions of Theorem 2.2, is a surjective linear isometry of $C^{\alpha}\left(X, x_{0}\right)$.

Now suppose that $T$ is a surjective isometry of $C^{\alpha}\left(X, x_{0}\right)$. Then the adjoint map $T^{*}$ is a surjective isometry of $C^{\alpha}\left(X, x_{0}\right)^{*}$ as well, and therefore $T^{*}$ is a bijection of the set of the extreme points of the unit ball of $C^{\alpha}\left(X, x_{0}\right)^{*}$. Moreover, as $T^{*}$ is a homeomorphism of $\left(C^{\alpha}\left(X, x_{0}\right)^{*}, w^{*}\right), T^{*}$ carries points which possess a $w^{*}$-metrizable neighbourhood in the $w^{*}$-closure of $F(W)$ into points with the same property.

Let $(x, y)$ be in $W$. By Lemmas 3.3 and $3.6, \widetilde{\delta}_{(x, y)}$ is an extreme point of the unit ball of $C^{\alpha}\left(X, x_{0}\right)^{*}$ which has a $w^{*}$-metrizable neighbourhood in the $w^{*}$-closure of $F(W)$. In view of the above comments, $T^{*}\left(\widetilde{\delta}_{(x, y)}\right)$ satisfies the same properties. Applying again Lemma 3.6, $T^{*}\left(\widetilde{\delta}_{(x, y)}\right)$ belongs to $F(W)$. Therefore there are $\sigma \in S_{\mathbb{K}}$ and $(u, v) \in W$ such that

$$
T^{*}\left(\widetilde{\delta}_{(x, y)}\right)=\sigma \widetilde{\delta}_{(u, v)}
$$


Let $X_{2}$ be the collection of all subsets of $X$ having exactly two elements. Clearly $T^{*}$ defines a bijection $\Phi: X_{2} \rightarrow X_{2}$ by

$$
\Phi(\{x, y\})=\operatorname{supp}\left(T^{*}\left(\widetilde{\delta}_{(x, y)}\right)\right) .
$$

This definition of $\Phi$ makes sense because if $\tau, \sigma \in S_{\mathbb{K}},(x, y),(u, v) \in W$ and $\tau \widetilde{\delta}_{(x, y)}=$ $\sigma \widetilde{\delta}_{(u, v)}$, then $\{x, y\}=\{u, v\}$. This follows as in Step 1 of Lemma 3.4.

There is a bijection $\varphi: X \rightarrow X$ such that $\Phi(\{x, y\})=\{\varphi(x), \varphi(y)\}$ for every $x, y \in X$ (see [1], Lemma 3). Plainly

$$
T^{*}\left(\widetilde{\delta}_{(x, y)}\right)=\sigma(x, y) \widetilde{\delta}_{(\varphi(x), \varphi(y))},
$$

where $\sigma(x, y) \in S_{\mathbb{K}}$. If for each $x$ in $X, \delta_{x}$ denotes the evaluation functional

$$
\delta_{x}(f)=f(x), \quad \forall f \in C^{\alpha}\left(X, x_{0}\right),
$$

we can write the preceding equality as

$$
T^{*}\left(\frac{\delta_{x}-\delta_{y}}{d^{\alpha}(x, y)}\right)=\sigma(x, y) \frac{\delta_{\varphi(x)}-\delta_{\varphi(y)}}{d^{\alpha}(\varphi(x), \varphi(y))} .
$$

Let us see that $\sigma(x, y) \frac{d^{\alpha}(x, y)}{d^{\alpha}(\varphi(x), \varphi(y))}$ does not depend on $x, y$. Let $z \notin\{x, y\}$. Then

$$
\begin{gathered}
\sigma(x, y) \frac{d^{\alpha}(x, y)}{d^{\alpha}(\varphi(x), \varphi(y))}\left(\delta_{\varphi(x)}-\delta_{\varphi(y)}\right)=T^{*}\left(\delta_{x}-\delta_{y}\right) \\
=T^{*}\left(\delta_{x}-\delta_{z}+\delta_{z}-\delta_{y}\right)=T^{*}\left(\delta_{x}-\delta_{z}\right)+T^{*}\left(\delta_{z}-\delta_{y}\right) \\
=\sigma(x, z) \frac{d^{\alpha}(x, z)}{d^{\alpha}(\varphi(x), \varphi(z))}\left(\delta_{\varphi(x)}-\delta_{\varphi(z)}\right)+\sigma(z, y) \frac{d^{\alpha}(z, y)}{d^{\alpha}(\varphi(z), \varphi(y))}\left(\delta_{\varphi(z)}-\delta_{\varphi(y)}\right),
\end{gathered}
$$

so that

$$
\sigma(x, y) \frac{d^{\alpha}(x, y)}{d^{\alpha}(\varphi(x), \varphi(y))}=\sigma(x, z) \frac{d^{\alpha}(x, z)}{d^{\alpha}(\varphi(x), \varphi(z))}=\sigma(z, y) \frac{d^{\alpha}(z, y)}{d^{\alpha}(\varphi(z), \varphi(y))} .
$$

Hence there exists a constant $k>0$ such that

$$
d(\varphi(x), \varphi(y))=k d(x, y)
$$

and clearly $\operatorname{diam}(\varphi(X))=k \operatorname{diam}(X)$. Since $\varphi$ is surjective, then $k=1$ and so

$$
d(\varphi(x), \varphi(y))=d(x, y)
$$

Hence $\varphi$ is an isometry of $X$. It follows that

$$
\sigma(x, y)=\sigma(x, z)=\sigma(z, y)
$$

and therefore there exists a $\tau \in S_{\mathbb{K}}$ such that $\sigma(x, y)=\tau$.

Finally, given $f \in C^{\alpha}(X)$ we have

$$
\begin{aligned}
\widetilde{\delta}_{(x, y)}(T(\rho(f)) & =T^{*}\left(\widetilde{\delta}_{(x, y)}\right)(\rho(f))=\tau \widetilde{\delta}_{(\varphi(x), \varphi(y))}(\rho(f))=\tau \frac{f(\varphi(x))-f(\varphi(y))}{d^{\alpha}(\varphi(x), \varphi(y))} \\
& =\frac{(\tau f \circ \varphi)(x)-(\tau f \circ \varphi)(y)}{d^{\alpha}(x, y)}=\widetilde{\delta}_{(x, y)}(\rho(\tau f \circ \varphi))
\end{aligned}
$$

for every $(x, y) \in W$. By Lemma 3.5 it follows that $\Phi(T(\rho(f)))=\Phi(\rho(\tau f \circ \varphi))$ for every extreme point $\Phi$ of the unit ball of $C^{\alpha}\left(X, x_{0}\right)^{*}$. The Krein-Milman Theorem implies that $\Phi(T(\rho(f)))=\Phi(\rho(\tau f \circ \varphi))$ for every $\Phi \in C^{\alpha}\left(X, x_{0}\right)^{*}$ and so

$$
T(\rho(f))=\rho(\tau f \circ \varphi) \text {. }
$$




\section{ACKNOWLEDGMENT}

The author thanks the referee for some valuable suggestions which improve this paper.

\section{REFERENCES}

[1] F. Cabello Sánchez, Diameter preserving linear maps and isometries, Arch. Math. 73 (1999), 373-379. MR 1712142(2000j:46047)

[2] F. González and V.V. Uspenskij, On homomorphisms of groups of integer-valued functions, Extracta Math. 14 (1999), 19-29. MR.1714426 (2000k:54016)

[3] M. Györy and L. Molnar, Diameter preserving linear bijections of $C(X)$, Arch. Math. 71 (1998), 301-310. MR1640086 (99h:46098)

[4] J. Johnson, Banach spaces of Lipschitz functions and vector valued Lipschitz functions, Trans. Amer. Math. Soc. 148 (1970), 147-169. MR0415289 (54:3379)

[5] J. Johnson, Lipschitz spaces, Pacific J. Math. 58 (2) (1975), 475-482. MR0385531 (52:6392)

[6] K. de Leeuw, Banach spaces of Lipschitz functions, Studia Math. 21 (1961), 55-66. MR0140927 (25:4341)

[7] C.K. Li and S. Pierce, Linear preservers problems, Amer. Math. Monthly 108 (2001), 591605. MR:1862098 (2002g:15005)

[8] C.K. Li and N.K. Tsing, Linear preserving problems: A brief introduction and some special techniques, Linear Algebra Appl. 162-164 (1992), 217-235. MR1148401 (93b:15003)

[9] E. Mayer-Wolf, Isometries between Banach spaces of Lipschitz functions, Israel J. Math. 38 (1981), 58-74. MR0599476 (82e:46036)

[10] D. Sherbert, The structure of ideals and point derivations in Banach algebras of Lipschitz functions, Trans. Amer. Math. Soc. 111 (1964), 240-272. MR0161177 (28:4385)

[11] A. Tromba, On the isometries of spaces of Hölder continuous functions, Studia Math. 57 (1976), 199-208. MR0420700 (54:8712)

[12] N. Weaver, Lipschitz Algebras, World Scientific, London, 1999. MR1832645 (2002g:46002)

Departamento de Álgebra y Análisis Matemático, Universidad de Almería, 04071, Almería, Spain

E-mail address: ajimenez@ual.es 